\title{
Structural studies of thermally stable, combustion- resistant polymer composites
}

\author{
Gregory N Smith ${ }^{1,2}$, James E Hallett ${ }^{1,3}$, Paul Joseph ${ }^{4}$, Svetlana Tretsiakova-McNally ${ }^{5}$, Tan Zhang ${ }^{6,7}$, \\ Frank D Blum ${ }^{6}$ and Julian Eastoe ${ }^{1}$
}

Composites of the industrially important polymer, poly(methyl methacrylate) (PMMA), were prepared by free-radical polymerization of MMA with varying amounts (1-30 wt. \%) of sodium dioctylsulfosuccinate (Aerosol OT or AOT) surfactant added to the reaction mixture. The composites with AOT incorporated show enhanced resistance to thermal degradation compared to pure PMMA homopolymer, and micro-cone combustion calorimetry measurements also show that the composites are combustionresistant. The physical properties of the polymers, particularly at low concentrations of surfactant, are not significantly modified by the incorporation of AOT, whereas the degradation is modified considerably for even the smallest concentration of AOT (1 wt. \%). Structural analyses over very different lengthscales were performed. X-ray scattering was used to determine $\mathrm{nm}$-scale structure, and scanning electron microscopy was used to determine $\mu \mathrm{m}$-scale structure. Two self-assembled species were observed: large phase-separated regions of AOT using electron microscopy and regions of hexagonally packed rods of AOT using $\mathrm{X}$-ray scattering. Therefore, the combustion resistance is observed whenever AOT self-assembles. These results demonstrate a promising method of physically incorporating a small organic molecule to obtain a highly thermally stable and combustionresistant material without significantly changing the properties of the polymer.

Polymer Journal (2017) 49, 711-719; doi:10.1038/pj.2017.44; published online 6 September 2017

\section{INTRODUCTION}

Poly(methyl methacrylate) (PMMA) is a very useful industrial polymeric material, mechanically rigid at ambient temperature and practically transparent. Its major drawback is that it is susceptible to oxidative thermal degradation at elevated temperatures. ${ }^{1,2}$ This can occur at temperatures as low as $170^{\circ} \mathrm{C}$, with a rate that increases with increasing at temperatures. ${ }^{3}$ Thermal decomposition is a known problem for many polymers, and therefore, the incorporation of inorganic materials can increase the degradation temperature. Many types of nanocomposite fillers have been reported in the literature, such as clays, silicates, graphite, carbon nanotubes, graphene and fumed silica. ${ }^{4-11}$ Nanomaterials, however, can possibly cause harm to biological systems, ${ }^{12}$ including the ability to enter cells, ${ }^{13}$ and combusting materials with nanoscale fillers raises possible environmental concerns. Entirely organic, thermally stable nanocomposites can overcome this limitation; their thermal degradation results only in small molecule products.

Surfactant molecules offer a promising alternative to these entirely inorganic, nanoscale fillers. As they are amphiphilic, possessing both polar and nonpolar portions, they are known to self-assemble into a variety of mesophases. ${ }^{14}$ It is, therefore, possible to use these self-assembled structures to template solid materials. In a series of pioneering studies, Texter et al. have studied the use of surfactant functional monomers as templates for composite materials. ${ }^{15}$ Nanostructures can be produced using polymerizable ionic monomers; ${ }^{16}$ however, this can be more simply achieved by adding a small molecule surfactant (sodium dioctylsulfosuccinate, Aerosol OT or AOT) during the polymer synthesis. ${ }^{17}$ The addition of small amounts of ionic monomers or surfactants results in composites with enhanced thermal stability.

Attaining a desirable material property such as this from the addition of a simple small molecule is remarkable, but it is not possible to understand or optimize this property without further study, as the structural and chemical origins are not yet clear. Only by determining the internal structure of the composite materials is it possible to develop structure-property relationships, which will enable more advanced, thermally stable and combustion-resistant materials to be developed. In this paper, composites of PMMA and AOT are synthesized, and the functional properties confirmed, using typical polymer characterization techniques, including thermogravimetric analysis (TGA) and micro-cone combustion calorimetry. The structural origin of the thermal properties of these composites has been

\footnotetext{
${ }^{1}$ School of Chemistry, University of Bristol, Cantock's Close, Bristol, UK; ${ }^{2}$ Department of Chemistry, University of Sheffield, Dainton Building, Brook Hill, Sheffield, South Yorkshire, UK; ${ }^{3} \mathrm{H}$.H. Wills Physics Laboratory, Tyndall Avenue, University of Bristol, Bristol, UK; ${ }^{4}$ Centre for Environmental Safety and Risk Engineering, Victoria University, Melbourne, Victoria, Australia; ${ }^{5}$ School of Pharmacy and Pharmaceutical Sciences, Ulster University, Coleraine, Co. Londonderry, Northern Ireland, UK and ${ }^{6}$ Department of Chemistry, Oklahoma State University, Stillwater, OK, USA

${ }^{7}$ Current address: Department of Materials Science and Engineering, Texas A\&M University, College Station, TX 77843, USA.

Correspondence: Dr GN Smith, Department of Chemistry, University of Sheffield, Dainton Building, Brook Hill, Sheffield, South Yorkshire S3 7HF, UK.

E-mail: g.n.smith@sheffield.ac.uk

Received 20 April 2017; revised 30 June 2017; accepted 5 July 2017; published online 6 September 2017
} 
determined using two techniques probing lengthscales over several orders of magnitude: scanning electron microscopy (SEM) to determine the $\mu \mathrm{m}$-scale structure and X-ray scattering to probe nm-scale domains. By combining multiple techniques to determine the distribution of surfactant in these materials, the relationship between internal structure and function has been revealed.

\section{EXPERIMENTAL PROCEDURES}

\section{Methods}

Ultraviolet-visible spectroscopy. Ultraviolet-visible spectra were measured using a Nicolet Evolution 300 Spectrophotometer (Thermo Electron Corp., UK) over a wavelength $(\lambda)$ range of 390-700 $\mathrm{nm}$. Composites were studied as synthesized in $2 \mathrm{~mm}$ pathlength quartz cells using an empty quartz cell as the background.

Gel permeation chromatography. Molecular weight distributions were assessed by gel permeation chromatography at $35^{\circ} \mathrm{C}$. The set-up comprised a guard column and two $5 \mu \mathrm{m}$ PL-gel Mixed-C columns connected in series to an Agilent Technologies 1260 Infinity refractive index detector, using tetrahydrofuran eluent containing 2.0 vol. \% triethylamine and 0.05 vol. \% butylhydroxytoluene at a flow rate of $1.0 \mathrm{ml} \mathrm{min}{ }^{-1}$. A series of 10 near-monodisperse PMMA standards ( $M_{p}$ ranging from 1280 to $330,000 \mathrm{~g} \mathrm{~mol}^{-1}$ ) were employed as calibration standards in conjunction with the above refractive index detector.

Differential scanning calorimetry. Temperature-modulated differential scanning calorimetry measurements were taken with a TA Instruments Q2000 TMDSC with nitrogen as a flow gas. The heating and cooling scans were taken from 35 to $160^{\circ} \mathrm{C}$, with a rate of $3{ }^{\circ} \mathrm{C} \mathrm{min}-1$, and a modulation amplitude of $\pm 1.0^{\circ} \mathrm{C}$ with a period of $60 \mathrm{~s}$.

Thermogravimetric analysis. Thermogravimetric analysis measurements were performed on $\sim 30-50 \mathrm{mg}$ samples using a TA Instruments Q50 TGA with a heating rate of $20^{\circ} \mathrm{C} \mathrm{min}^{-1}$ in an air atmosphere.

$X$-ray scattering. X-ray scattering measurements were performed using a GANESHA 300 XL (SAXSLAB, Denmark) system with an adjustable sampledetector distance. Small-angle (SAXS), medium-angle (MAXS) and wide-angle (WAXS) measurements were performed with sample to detector distances of 1050,450 and $100 \mathrm{~mm}$, respectively. X-rays were detected using an in-vacuum PILATUS $300 \mathrm{k}$ (Dectris, Switzerland) detector and were generated using a sealed tube generator with a $\mathrm{Cu}$ anode (X-ray wavelength $1.54 \AA$ ). Fluid samples were loaded into $1 \mathrm{~mm}$ borosilicate glass capillary cells tubes. For a typical experiment, measurements were performed for $10 \mathrm{~min}$. A transmissionnormalized empty cell background was subtracted from the data, and the beamstop and any bad sectors on the detector were masked. The data were then radially averaged to produce one-dimensional scattering curves for further analysis. Data have been fit using either the SASfit ${ }^{18,19}$ or the SasView ${ }^{20}$ small- $^{-}$ angle scattering software packages.

Pyrolysis combustion flow calorimetry. Pyrolysis combustion flow calorimetry (PCFC), also known as micro-scale combustion calorimetry, has been shown to be a very valuable small-scale technique for screening flammability of different materials in recent years. ${ }^{21}$ PCFC is an ideal tool for these studies as a rapid screening technique since it only requires a few milligrams of polymer for testing and often provides a wealth of combustion-related data. PCFC works on a principle of oxygen depletion calorimetry, relating to Hugget's principle that $1 \mathrm{~kg}$ of consumed oxygen corresponds to $13.1 \mathrm{MJ}$ of released energy for any organic material. At first, a polymeric sample is rapidly heated to a state of controlled pyrolysis in an inert atmosphere of nitrogen (method A: anaerobic conditions) or in a mixture of nitrogen and oxygen (method B: aerobic conditions), followed by a rapid oxidation at high temperatures (combustion) of the pyrolysate in an excess of oxygen. In the present study, the operational conditions were in conformance with the test method A. This method also is an established ASTM standard for testing flammability characteristics of solid materials.

Measurements were performed using a Fire Testing Technology Ltd. (Gosport, UK) micro-scale combustion calorimeter to study the combustion properties. The description of this method including the operating parameters is published in detail elsewhere. ${ }^{22}$ For each run, accurately weighed ( $10 \mathrm{mg}$ ) and finely powdered samples were first heated to about $900{ }^{\circ} \mathrm{C}$ at a heating rate of $\sim 0.9^{\circ} \mathrm{C} \mathrm{s}^{-1}$, in a stream of nitrogen flowing at a rate of $80 \mathrm{~cm}^{3} \mathrm{~min}^{-1}$. The volatile thermal degradation products, thus obtained, were then mixed with a stream of pure oxygen (at a flow rate of $20 \mathrm{~cm}^{3} \mathrm{~min}^{-1}$ ) prior to entering a combustion chamber maintained at $900{ }^{\circ} \mathrm{C}$. Each sample was run in triplicate.

\section{Materials}

The composites are prepared from the free-radical polymerization of methyl methacrylate (MMA) with added sodium dioctylsulfosuccinate (Aerosol OT or AOT), using the method of Ge and Texter. ${ }^{17}$ The radical initiator azobisisobutyronitrile (AIBN, 98\%, Acros Organics, UK) at a concentration of $1 \mathrm{wt.} \%$ and AOT (98\%, Aldrich, UK) at concentrations ranging from 1 to $30 \mathrm{wt} . \%$ were dissolved in MMA (99\%, inhibitor removed, Aldrich, UK) to form clear solutions. The solutions were then placed in $5 \mathrm{~mm}$ inner diameter NMR tubes and immersed into an oil bath preheated to $70^{\circ} \mathrm{C}$. The synthesis of samples for X-ray scattering and ultraviolet-visible spectroscopy was identical, except the reactions were performed in containers appropriate for the technique ( $1 \mathrm{~mm}$ diameter borosilicate glass capillaries for X-ray scattering and $2 \mathrm{~mm}$ thick quartz cells for ultraviolet-visible spectroscopy).

\section{RESULTS AND DISCUSSION}

The potential of using these surfactant-containing composites as replacements for PMMA homopolymer will be considered first. An image of the PMMA-AOT composites is shown in Figure 1a. Adding surfactant to the polymerization mixture increases the turbidity. The pure PMMA sample is optically transparent, and the turbidity then increases with increasing surfactant concentration. The transparency of PMMA is key to many of its industrial uses. ${ }^{1,2}$ The turbidity of the composites was assessed using ultraviolet-visible spectroscopy over the visible range ( $\lambda$ from 390 to $700 \mathrm{~nm}$ ), and the spectra are shown in Figure $1 \mathrm{~b}$. Without any surfactant additive, the PMMA samples are highly transparent $(\sim 85 \%)$, as expected given the applications of the polymer. With a small amount of AOT incorporated (1 wt. \%), the transparency is decreased slightly. At even higher AOT concentrations, the composites are essentially opaque. The transmittance of the PMMA-AOT composites not only varies between the different samples but is also wavelength dependent. This may be explained if AOT selfassembles into colloidal aggregates in the material, as species of this size are known to scatter light due to the Tyndall effect. ${ }^{23-26}$

The addition of surfactant changes more than just the visual appearance of the polymer; it also modifies the physical properties of the material itself. This has consequences for the polymers, as seen in gel permeation chromatography analysis of the four polymers shown in Figure 2 and Table 1. As expected from a bulk radical polymerization reaction, the polymerization is poorly controlled, ${ }^{27}$ with a bimodal molar mass $\left(M_{w}\right)$ distribution and a large molar mass dispersity $\left(\bigoplus_{M}\right)$. The sample containing $1 \mathrm{wt}$. \% AOT has a molar mass distribution similar to the pure PMMA sample, indicating that this small amount of surfactant does not influence the polymerization reaction. At higher concentrations, the incorporation of AOT seemingly impacts the propagation or termination of the reaction, resulting in a different molar mass distribution. Larger molar mass polymers are increasingly favored. This does not influence the $M_{n}$, which is constant for the samples, but it does increase the $M_{w}$ and consequently $\bigoplus_{M}$. Introducing surfactant to the system, therefore, also has some influence on the composite material, although it is minimal. The glass transition temperature $\left(T_{g}\right)$ of the composites has also been measured using temperature-modulated differential scanning calorimetry, and the thermograms are shown in Supplementary Figure S1. The peak maximum in the derivative of the reversible heat flow is taken as the value of the $T_{g}$, consistent with literature on PMMA-containing materials. ${ }^{28} T_{\mathrm{g}}$ for PMMA-AOT composites is the 
a
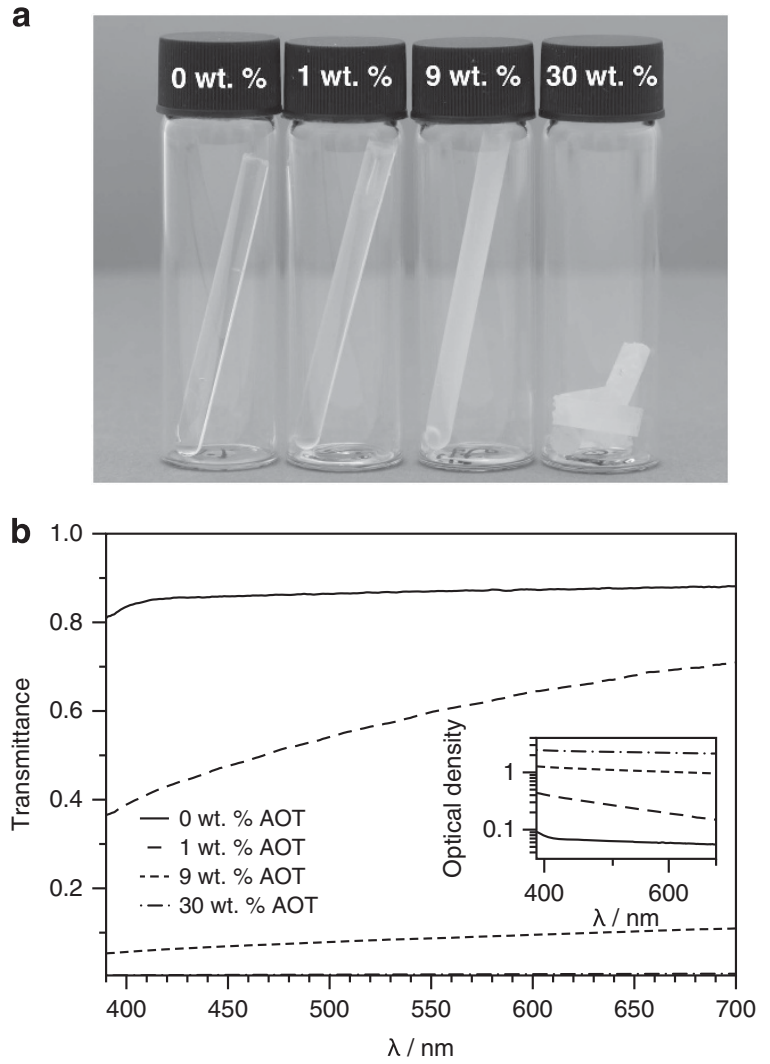

Figure 1 Optical properties of PMMA-AOT composites. (a) Optical image of PMMA-AOT composites ( $0,1,9$, and 30 wt. \% AOT, left to right). (b) Transmittance of visible light through PMMA-AOT composites as a function of wavelength ( $\lambda$ from 390 to $700 \mathrm{~nm}$ ). The curve for the sample containing 30 wt. \% AOT is along the axis of the plot. The inset shows optical density as a function of wavelength $\lambda$, required for quantitative analysis of Tyndall scattering. ${ }^{23-26}$ PMMA without any additive is highly transparent, and the addition of a small amount of AOT (1 wt. \%) decreases the transparency slightly. Composites with higher concentrations of AOT are nearly opaque. A full colour version of this figure is available at the Polymer Journal journal online.

same as for the PMMA homopolymer, demonstrating that the incorporation of AOT does not influence certain properties of the materials. Although the molar masses increase with the amount of added surfactant, the large molar mass of the polymer means that this makes little difference to the $T_{g}{ }^{29}$

The most striking effect of introducing surfactant into a polymeric material is the modification of their thermal stability. PMMA-AOTbased composite materials (in free or polymerizable forms) are resistant to combustion and thermally stable. ${ }^{16,17}$ To understand the role of a small molecule surfactant, the internal structure of the composites has been determined using electron microscopy and X-ray scattering. These methods are ideally suited to such analysis as the sodium cation of the AOT surfactant has a moderate $Z$-number, providing an inherent electron-density difference. The thermal properties of the materials will be discussed first, and then the structural properties will be considered.

\section{Degradation analysis}

The rate of thermal degradation is the significant difference between composites either with or without added AOT. This was quantified using TGA, and the results are shown in Figure 3. The data are shown in two formats: the fraction of polymer that has degraded $(\alpha)$ as a

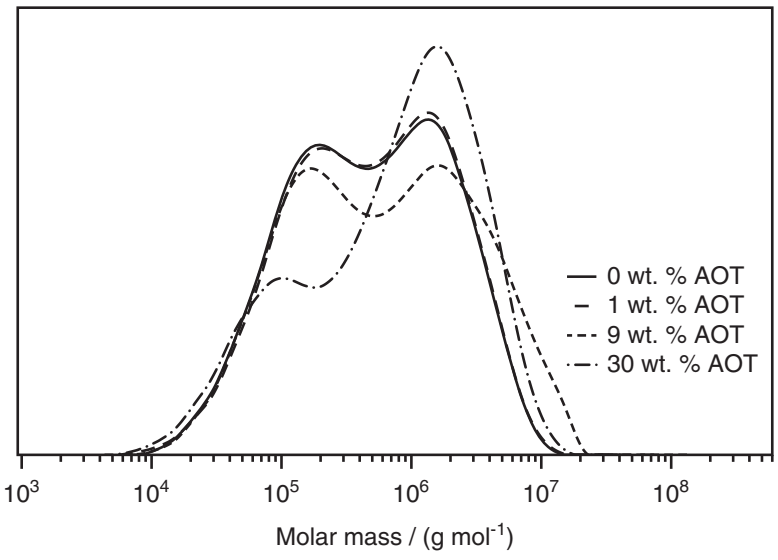

Figure 2 GPC chromatograms of PMMA-AOT composites in THF against PMMA standards. As the polymerization is not controlled, the molar mass distributions are broad. The pure PMMA and the $1 \mathrm{wt}$. \% AOT sample are very similar; a small amount of surfactant does not impact the polymerization reaction. The 9 and $30 \mathrm{wt}$. \% AOT samples are different; the distribution is shifted to larger species for both. GPC, gel permeation chromatography. A full colour version of this figure is available at the Polymer Journal journal online.

function of temperature (T) in Figure $3 \mathrm{a}$ and the first derivative of $\alpha$ with respect to temperature $(\mathrm{d} \alpha / \mathrm{d} T)$ in Figure $3 \mathrm{~b}$. These two representations emphasize different points about the data. In Figure 3a, it can be seen that moderate amounts of AOT (1 and 9 wt. \%) shift the entire decomposition of the polymer to higher temperatures. The degradation of the composite with the highest concentration of surfactant (30 wt. \%) begins at a lower temperature than for PMMA, but this due to the degradation of the AOT surfactant itself, which occurs at $\sim 300^{\circ} \mathrm{C} .{ }^{30}$ In Figure $3 \mathrm{~b}$, the differences between the surfactant-containing composites and the PMMA homopolymer are more evident. The high temperature peak, representing the temperature where the rate of degradation is greatest, is shifted to even higher temperatures when surfactant is present by $\sim 35^{\circ} \mathrm{C}$. This clearly shows that the composites are more thermally stable with incorporated AOT.

The derivative of the TGA data is particularly informative for understanding how the degradation reaction proceeds. The time dependence of the rate constant $(\mathrm{d} \alpha / \mathrm{d} t)$ of thermal analysis data can be described by an Arrhenius-like expression, where $\alpha$ is the fraction of the product consumed, $n$ is the order of the reaction, $E$ is the activation energy, and $T$ is the temperature. ${ }^{31}$

$$
\frac{\mathrm{d} \alpha}{\mathrm{d} t}=A(1-\alpha)^{n} \mathrm{e}^{-E / R T}
$$

At a constant heating rate $(\phi)$, the time derivative $(\mathrm{d} \alpha / \mathrm{d} t)$ can be expressed in terms of the temperature derivative $(\mathrm{d} \alpha / \mathrm{d} T)$.

$$
\frac{\mathrm{d} \alpha}{\mathrm{d} T}=\frac{A}{\phi}(1-\alpha)^{n} \mathrm{e}^{-E / R T}
$$

This equation is sufficient to describe thermal degradation that occurs as a single event, but if the sample degrades in multiple steps, the overall rate of conversion can be modeled as a sum of independent steps. All parameters are the same as in equations (1) and (2) except 
Table 1 Polymer characterization of PMMA-AOT composites

\begin{tabular}{lcccc}
\hline [AOT] $/$ wt. \% & $M_{n} /\left(10^{5} \mathrm{~g} \mathrm{~mol}^{-1}\right)$ & $M_{w} /\left(10^{5} \mathrm{~g} \mathrm{~mol}^{-1}\right)$ & $\Xi_{M}\left(M_{w} / M_{n}\right)$ & $T_{g} /{ }^{\circ} \mathrm{C}$ \\
\hline 0 & 1.83 & 11.1 & 6.10 & 117 \\
1 & 1.83 & 11.4 & 6.23 & 115 \\
9 & 1.91 & 18.0 & 9.39 & 115 \\
30 & 1.82 & 15.5 & 8.52 & 117 \\
\hline
\end{tabular}

Abbreviations: AOT, Aerosol OT; PMMA, poly(methyl methacrylate).

for $r_{i}$, which is the yield coefficient of the ith step $\left(\Sigma_{i} r_{i}=1\right){ }^{32}$

$$
\begin{gathered}
\frac{\mathrm{d} \alpha}{\mathrm{d} t}=\phi \frac{\mathrm{d} \alpha}{\mathrm{d} T}= \\
\sum_{i} r_{i}\left[A_{i} \cdot \exp \left(-\frac{E_{i}}{R T}\right) \cdot\left[1-\left(1-n_{i}\right) \cdot \frac{A_{i} R T^{2}}{\phi\left(E_{i}+2 R T\right)} \cdot \exp \left(-\frac{E_{i}}{R T}\right)\right]^{\frac{n_{i}}{1-n_{i}}}\right]
\end{gathered}
$$

The thermal degradation of PMMA has been well-studied in the literature. ${ }^{32-37}$ In particular, Manring et al. studied the degradation of PMMA in the 1980s and 1990s using chains with functionality incorporated. ${ }^{3-36}$ PMMA degradation can be modeled using equation (4) using four steps. ${ }^{32}$ The highest temperature peak corresponds to random scission, and this is the primary degradation mechanism of interest for these PMMA-AOT composites. The molar mass dispersity of these free-radical polymerized polymers $\left(\bigoplus_{M}\right.$, Table 1) is large, giving polymers with a broad distribution of degrees of polymerization. Degradation of polymers can be influenced by the length of the chain, ${ }^{33}$ and therefore, the random scission cannot be fit using a single peak as done by Ferriol et al. ${ }^{32}$ and rather must be fit using two peaks. A comparison between the degradation of the PMMA homopolymer measured in this study and that measured by Ferriol et al. ${ }^{32}$ is shown Supplementary Figure S2.

Modeling the kinetics of the decomposition reaction can reveal the differences between the samples. The degradation of the PMMA homopolymer and the composite containing $1 \mathrm{wt}$ \% AOT have been fit using equation (4). The best fit parameters are shown in Supplementary Table S1. These two samples provide an interesting comparison as the properties of the polymer itself (Table 1 and Figure 3) are very similar whereas the thermal degradation of the materials is considerably different (Figure 3 ).

The three low temperature peaks are similar between the two samples shown in Figure 4. The degradation is approximately $30-35 \%$ complete (calculated from a sum of $r_{i}$ for the three steps) in these first three steps for both samples. The magnitude of $E$ is reduced for the $1 \mathrm{wt}$. \% AOT composites, however (from $E_{2}=119.3 \mathrm{~kJ} \mathrm{~mol}^{-1}$ and $E_{3}=202.3 \mathrm{~kJ} \mathrm{~mol}^{-1}$ for 0 wt. \% AOT to $E_{2}=85.9 \mathrm{~kJ} \mathrm{~mol}^{-1}$ and $E_{3}=144.2 \mathrm{~kJ} \mathrm{~mol}^{-1}$ for $1 \mathrm{wt}$ \% AOT). This is perhaps due to a small contribution from the inclusion of AOT, which thermally decomposes at a lower temperature than PMMA. ${ }^{30}$ The interesting differences, relating to the increased thermal stability, can be seen in the two high temperature peaks, which correspond to random chain scission. ${ }^{32}$ The difference in activation energy is the most revealing difference between the two samples. $E_{5}$ of the highest temperature peak in the degradation of the PMMA homopolymer $\left(198.6 \mathrm{~kJ} \mathrm{~mol}^{-1}\right)$ agrees well with recent studies of PMMA degradation, which give values of $\sim 200 \mathrm{~kJ} \mathrm{~mol}^{-1}$. 33,37 The reaction orders $\left(n_{4}\right.$ and $n_{5}$ between 1.0 and 1.6) and yield coefficients $\left(r_{4}+r_{5} \approx 0.6\right)$ for the final steps of both the 0 and 1 wt. \% samples are essentially the same. The activation energies, however, are greater for the highest a

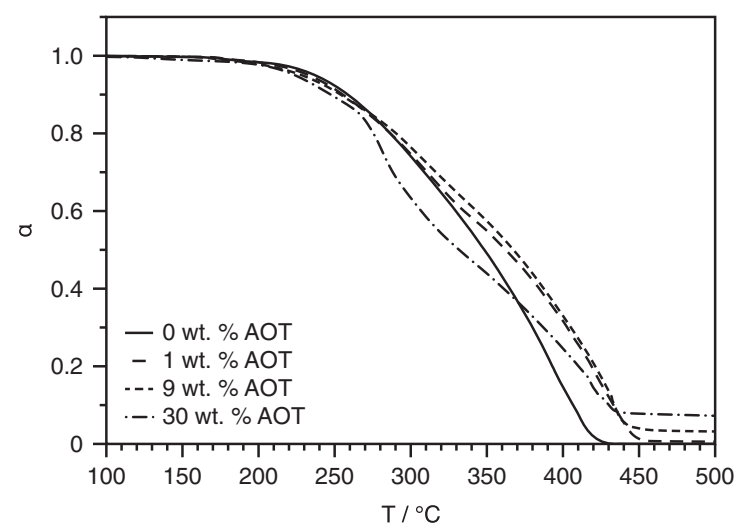

Weight loss as a function of temperature.

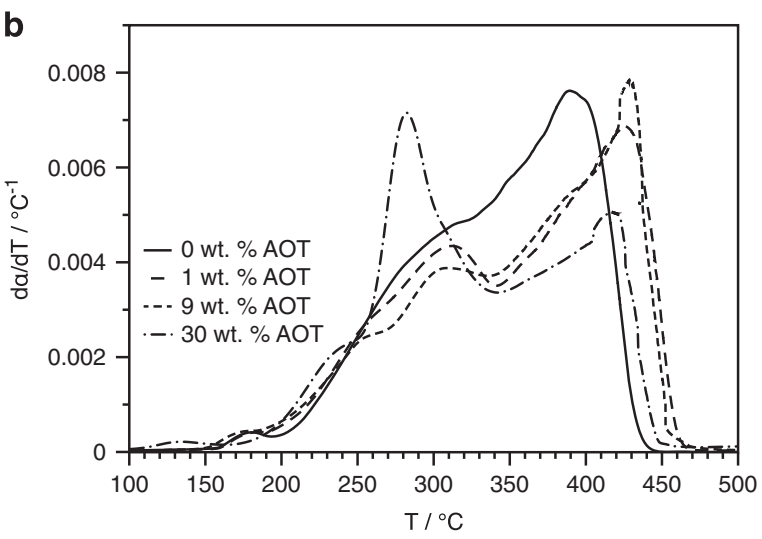

Derivative of weight loss with temperature.

Figure 3 TGA data of PMMA-AOT composites. (a) shows the data in the typical format (the fraction consumed $\alpha$ as a function of temperature $T$ ), and this shows that the incorporation of AOT into the composites (even at 1 wt. \%) is sufficient to impart thermal stability to the materials. (b) shows the data as the first derivative of mass loss with concentration ( $d \alpha / \mathrm{d} T$ ). A full colour version of this figure is available at the Polymer Journal journal online.

temperature degradation steps of the AOT-containing composite. For the PMMA homopolymer, $E_{4}=108.0 \mathrm{~kJ} \mathrm{~mol}^{-1}$ and $E_{5}=198.6 \mathrm{~kJ} \mathrm{~mol}^{-1}$; for the 1 wt. \% AOT composite, $E_{4}=115.7 \mathrm{~kJ} \mathrm{~mol}^{-1}$ and $E_{5}=205.1 \mathrm{~kJ} \mathrm{~mol}^{-1}$. The difference upon adding AOT is the same for both steps, approximately $7 \mathrm{~kJ} \mathrm{~mol}^{-1}$. This appears to be the amount of energy that is required to overcome the thermal stability imparted by the AOT surfactant. That the degradation of the composites can be influenced by such a small amount of surfactant is remarkable and shows a tangible influence of the incorporation of surfactant.

In addition to the thermogravimetry measurements of polymer degradation, the following parameters were measured using PCFC: peak heat release rate ( $\mathrm{pHRR}$ ), temperature at peak heat release rate (Temp. to pHRR), total heat release (THR), heat release capacity (HRC), and percentages of the char residues.

The data obtained and presented in Table 2 are average values of three measurements, with the corresponding standard deviation given as the error. The values for all measurements along with the correlation coefficient (CC) are shown in Supplementary Table S2.

The values of HRC (maximum amount of heat released per unit mass per degree temperature) can serve as a reliable indicator of the 


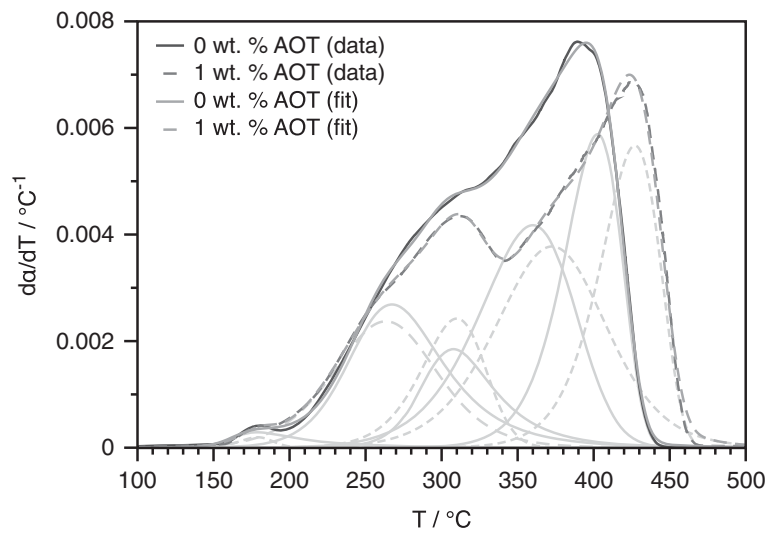

Figure 4 The derivative of the TGA data $(\mathrm{d} \alpha / \mathrm{d} T)$ as a function of temperature for PMMA homopolymer and the $1 \mathrm{wt}$. \% AOT composite fit to equation (4). The data for both can be fit using a five-step degradation. The first three steps are similar, but the final two steps at highest temperatures, plausibly corresponding to random chain scission, are shifted to higher temperatures for the AOT-containing composite. A full colour version of this figure is available at the Polymer Journal journal online.

Table 2 Values of some relevant parameters from PCFC runs

\begin{tabular}{lclr}
\hline [AOT]/Wt. \% & Temperature to $p H R R /{ }^{\circ} \mathrm{C}$ & pHRR/( $\left.\mathrm{Wg}^{-1}\right)$ & \multicolumn{1}{c}{ THR/(KJ $\left.\mathrm{g}^{-1}\right)$} \\
\hline 0 & $384 \pm 0.82$ & $338 \pm 9.42$ & $24.07 \pm 1.01$ \\
1 & $385 \pm 0.58$ & $337 \pm 8.52$ & $22.80 \pm 0.62$ \\
9 & $388 \pm 0.58$ & $302 \pm 13.53$ & $22.17 \pm 0.09$ \\
30 & $387 \pm 1.63$ & $207 \pm 7.33$ & $21.8 \pm 0.26$ \\
\hline
\end{tabular}

\begin{tabular}{lcc}
{$[$ AOT] $/$ wt. \% } & HRC/(J g $\left.{ }^{-1} K^{-1}\right)$ & Char yield/wt. \% \\
\hline 0 & $334 \pm 8.58$ & $2.30 \pm 2.01$ \\
1 & $331 \pm 5.45$ & $1.65 \pm 2.33$ \\
9 & $229 \pm 14.34$ & $3.6 \pm 4.45$ \\
30 & $205 \pm 7.33$ & $6.7 \pm 1.52$
\end{tabular}

Abbreviations: AOT, Aerosol OT; HRC, heat release capacity; PCFC, pyrolysis combustion flow calorimetry; pHRR, peak heat release rate; THR, total heat release.

flammability of a polymer. ${ }^{38}$ The instrument also recorded plots of the heat release rate (HRR) versus temperature, shown in Figure 5.

It can be observed that differences in the profiles of the thermograms of the samples, as revealed through the TGA experiments, are also reflected in some of the parameters obtained by performing the PCFC measurements. Generally, the values obtained for the relevant experimental parameters, such as pHHR, THR and heat release capacity, clearly indicate that the PMMA-AOT composites are more fire retarded than the unmodified counterpart. The stabilizing effect of AOT in the samples, as reflected through the enhancements in the on-set temperature of degradation in TGA traces, is also nominally evident in the pHHR values; however, there is obviously a profound diminishing return in the case of the sample with the high percentage of AOT ( 30 wt. \%), as the degradation of AOT in this case is bound to dominate. A similar trend is evident with regard to the values of THR. However, the most striking feature and an unequivocal evidence pertaining to the enhancement of the combustion resistance of the samples can be observed in both the values of peak heat release rate and HRC, the former being the most reliable indicator of the overall fire hazard of a material when present as a part of a fuel loads in enclosures. The amount of char yields also reveals an upward trend, albeit not uniform (with

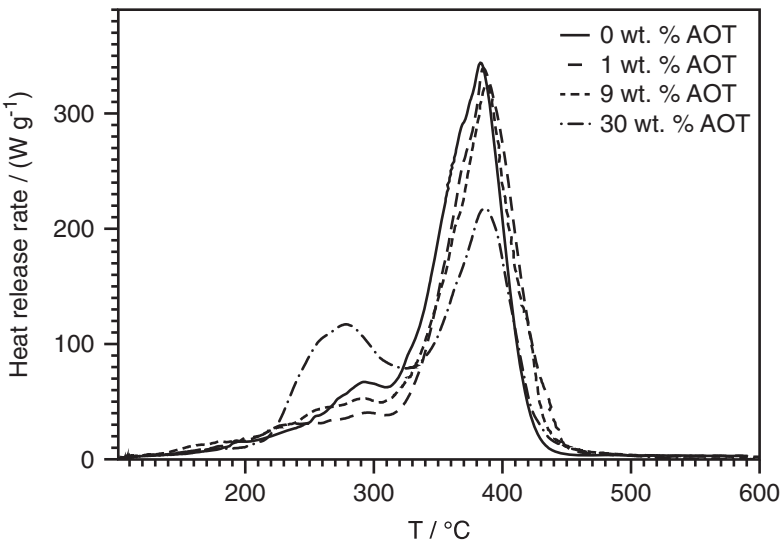

Figure 5 Representative plots of heat release rate versus temperature for each of the AOT-containing composites. A full colour version of this figure is available at the Polymer Journal journal online.

relatively high values for the Correlation Coefficient), and can be also regarded as an indication of decreased combustibility of the samples. This is in agreement with the TGA runs in air, an oxidative atmosphere, whereas during PCFC measurements, the initial degradation was accomplished under nitrogen, an anaerobic condition. The amount of residue remaining at the end of the TGA runs (Figure 3a) increases with increasing concentration of AOT. Below 30 wt. \% AOT, the amount of char in the PCFC measurements is hard to resolve, but the amount of residue remaining in both the TGA measurements at $500{ }^{\circ} \mathrm{C}(7.2 \%)$ and the PCFC measurements $(6.7 \pm 1.52 \%)$ is very similar.

\section{Structural analysis}

There is clearly a difference in the structures present in the composites; this can be seen by comparing the appearance of the composites shown in Figure 1a. Ge and Texter mention the presence of $\mu \mathrm{m}$-scale hemispheres, ${ }^{17}$ which they suggest are most likely to be vesicular, due to the well-known ability of AOT to form lamellar structures. ${ }^{39,40}$ Objects of this size could easily give rise to sufficient light scattering to make these materials turbid, and this is indeed seen in Figure 1b. These hemispheres have been studied using SEM, and the typical images are shown in Figure 6. The hemispheres only seem to be present in the more concentrated samples (9 and $30 \mathrm{wt}$. \% AOT, Figure $6 \mathrm{~b}$ and c). The most dilute sample ( 1 wt. \% AOT, Figure $6 \mathrm{a}$ ) is smooth and devoid of hemispherical regions. The most concentrated sample (30 wt. \% AOT, Figure 6c) appears to consist of hemispheres connected by films of surfactant. Given the intermediate structure of the composite containing 9 wt. \% AOT, energy dispersive X-ray analysis (EDX) was performed on two regions in the composite to determine atomic composition: a smooth region of the composite (Region 1) and one of the hemispheres (Region 2). These regions are labeled in Figure 6b. The EDX results (Figure 6d and e) clearly show that the smooth region is devoid of surfactant but that the hemisphere contains surfactant, due to peaks from sodium and sulfur. The size of the circled aggregate in Figure $6 \mathrm{~b}$ is $6.6 \mu \mathrm{m}$ in diameter, agreeing with the hemispheres identified by Ge and Texter. ${ }^{17}$ Aggregates of this size are also consistent with the turbidity data shown in Figure $1 \mathrm{~b}$. The size of colloids can be determined from Tyndall scattering data by analyzing the dependence of the optical density as a function of wavelength, using the method of Heller. ${ }^{25,26,41}$ Using the optical density from $400 \geqslant \lambda \geqslant 600 \mathrm{~nm}$ and making two assumptions (that the relationship between wavelength exponent and size continues past Heller's limits ${ }^{41}$ and that the analysis is the same for an assumed 
a

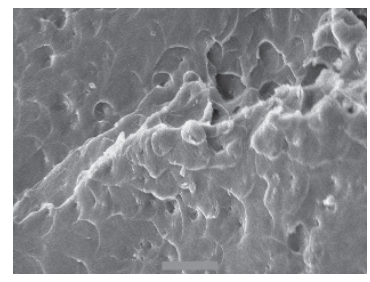

$1 \%$ AOT. b

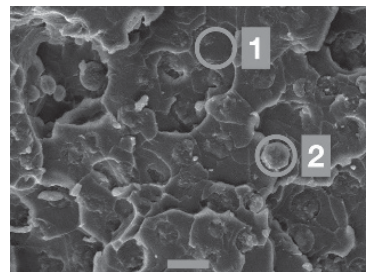

$9 \%$ AOT.
C

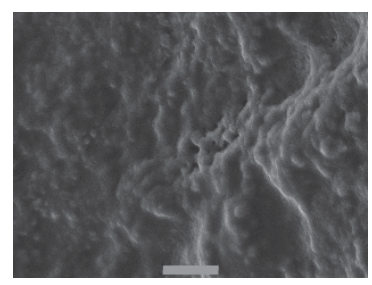

$30 \%$ AOT.

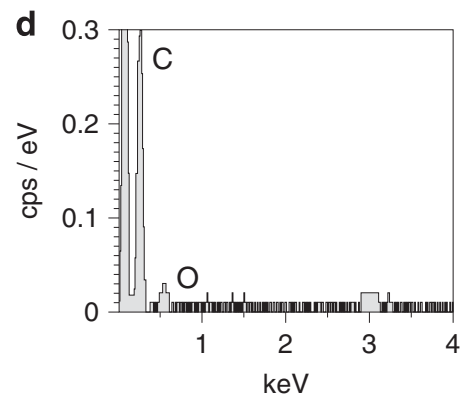

$9 \%$ AOT (Region 1).

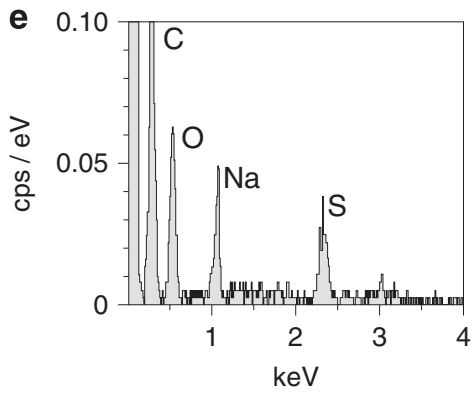

$9 \%$ AOT (Region 2).

Figure 6 SEM micrographs (a-c) of PMMA-AOT composites with different concentrations of AOT and EDX spectra (d and e) of surfactant-free and surfactantrich regions of the PMMA-AOT composite with 9 wt. \% AOT. The regions that the EDX spectra were taken from are shown in (b). Scale bars in SEM micrographs denote $10 \mu \mathrm{m}$. A full colour version of this figure is available at the Polymer Journal journal online.

refractive index ratio of 1.05 as the actual PMMA/AOT refractive index ratio of $1.01^{42,43}$ ), the size of the scattering domains in the 9 and 30 wt. \% AOT composites is $\sim 6-7 \mu \mathrm{m}$ in diameter. Both analyses show that large aggregates are present. From SEM alone it is not possible to identify the exact morphology of these structures (vesicular or spherical); however, it is clear that in samples with more AOT there are extended, surfactant-rich regions.

Electron microscopy is only sensitive to structures with features greater than $>10 \mathrm{~nm},{ }^{44}$ but surfactants can also self-assemble in solution into much smaller ( $\AA$ to $\mathrm{nm}$ ) aggregates. Real-space techniques, such as microscopy, are unable to distinguish features at these lengthscales, but inverse-space techniques, such as small-angle scattering, are appropriate methods. X-ray scattering is particularly useful for these composites, as it is sensitive to differences in electron density. A combination of small-, medium-, and wide-angle X-ray scattering (SAXS/MAXS/WAXS) measurements were performed on PMMAAOT composites synthesized in borosilicate glass capillaries, and the processed scattering curves are shown in Figure 7. Measurements could not be performed on pure PMMA alone, as the reaction repeatedly caused the capillaries to break. Measurements were also performed on anhydrous AOT alone to enable a comparison between its internal structure and the self-assembled structures in the composites.

The scattering data from the solid AOT surfactant alone have been measured and interpreted previously, as arising from hexagonal rods with an inverse structure (polar groups in the center). ${ }^{45,46}$ The measurement in this study (Figure 7 ) agrees with this interpretation. The primary peak occurs at $Q=0.3 \AA^{-1}$, which corresponds to a $d$-spacing of $20.5 \AA$ or, equivalently, a core radius of $11.8 \AA$. In contrast to these previous reports, ${ }^{45,46}$ a small peak at a peak ratio of $\sqrt{3}\left(Q=0.53 \AA^{-1}\right)$ is also observed, supporting the hexagonal phase structure. The other features in the scattering of the AOT solid are due to either the non-crystalline, liquid-like arrangement of the surfactant alkyl tails (peak at high-Q) ${ }^{45,47}$ or smooth interfaces (scattering proportional to $Q^{-4}$ at low- $Q$ ). That no power law proportional to $Q^{-1}$, as expected for rigid rods, ${ }^{48}$ is observed in the $Q$-range measured demonstrates that these smooth objects have a large diameter.

Having interpreted the scattering from the AOT alone, the scattering from the PMMA-AOT composites can be compared and contrasted to it. The scattering at high- $Q$ differs slightly, now consisting of two broad peaks at $\sim 1 \AA^{-1}$ and $\sim 2 \AA^{-1}$, as observed previously for WAXS measurements on PMMA. ${ }^{49}$ The scattering at low- $Q$ is similar, a featureless power law in $Q$. The power law exponent (Porod slope) between 3 and 4 is obtained for the scattering in the low- $Q$ region, indicating that these large objects have a rough, fractal interface. ${ }^{50}$ The concentration of these seems to differ, as the intensity of the power law scattering from the $1 \mathrm{wt}$ \% AOT composite is less than the others. This also seems to rule out vesicular AOT self-assembled structures, at least ones with a thin wall thickness. It would be possible for the scattering data to be consistent with the surface of vesicles, but the shell thickness should clearly be observable in the Q-range measured (AOT lamella have thicknesses of $28 \AA^{51}$ ). From the X-ray scattering data, the large structures seem most likely to be poorly defined spheres, given that $\mu \mathrm{m}$-scale features were observed by SEM and EDX (Figure 6).

The sharp peaks at $Q \approx 0.3 \AA^{-1}$ in all of the PMMA-AOT composites are likely to be the most important structures for understanding the thermal stability of the materials. The peaks occur at precisely the same $Q$ as for the AOT solid (shown in Figure 7 by the vertical dashed line), consistent with hexagonally packed rods being present in the PMMA-AOT composites as well as the AOT solid. As the intensity of the peaks in the 9 wt. $\%$ and 30 wt. \% AOT composites is the same, these self-assembled structures must have reached a solubility limit below $9 \mathrm{wt}$. \%, and the fact that the $1 \mathrm{wt}$. \% composites are thermally stable shows that only a small amount of surfactant is required to reach achieve this property.

This naturally suggests a question about the origin of these hexagonal species. Are they due to AOT insolubility, or are they due 

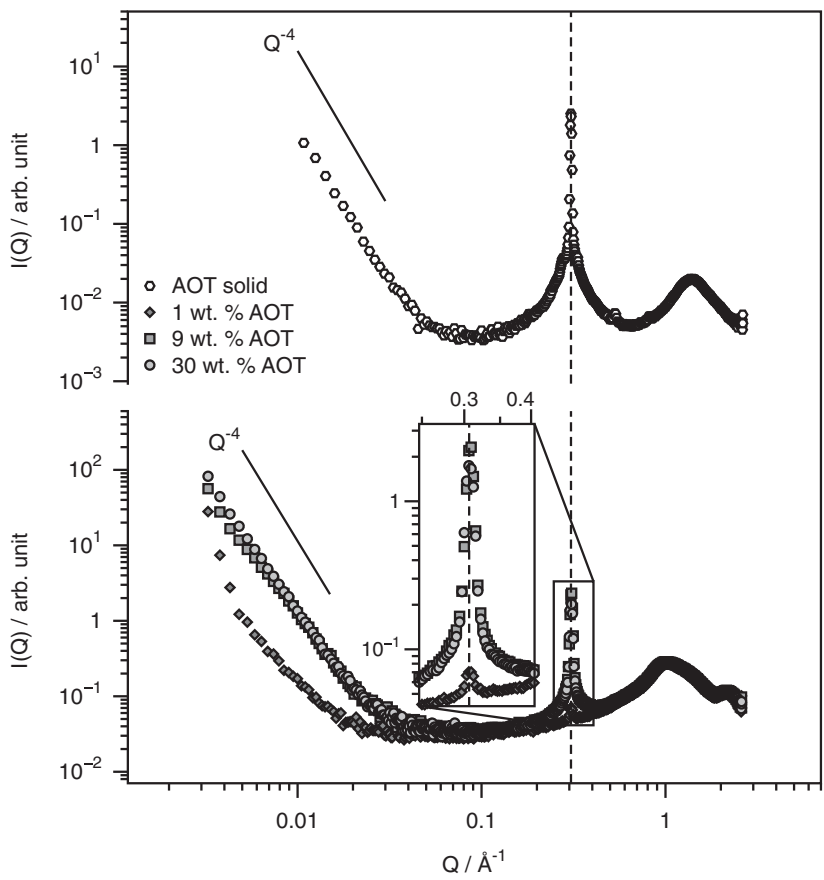

Figure 7 X-ray scattering data of anhydrous AOT solid (top) as well as PMMA-AOT composites at several AOT concentrations (bottom). Both sets of data consist of three regions: scattering proportional to $Q^{-4}$ at low- $Q$ due to long-ranged smooth interfaces, a peak at $Q=0.3 \AA^{-1}$ due to a reverse hexagonal phase, and peaks at high- $Q$ due to short-ranged liquid-like order. The peak at $Q=0.3 \AA^{-1}$ in all the samples shows that the AOT phase separates into reverse hexagonal phase regions in the PMMA-AOT composites. The intensity of the scattering from the peaks in the PMMA-AOT composites shows that there is a lower concentration of self-assembled AOT in the $1 \mathrm{wt}$ \% composite, as would be expected, but that there is the same amount of self-assembled AOT in the 9 and $30 \mathrm{wt}$. \% composites. A full colour version of this figure is available at the Polymer Journal journal online.

to phase-separation during the polymerization process? To study this, MAXS/WAXS measurements were made on samples of AOT surfactant dispersed in the MMA monomer. These should be equivalent to the systems at the start of the reaction. The scattering data are shown in Figure 8. Qualitatively the scattering from the three samples is very similar. All have a peak at high- $Q$, due to the arrangement of alkyl tails as also seen for the AOT solid in Figure $7{ }^{45,47}$ A peak at this $Q$ agrees with literature WAXS measurements on AOT and AOT-stabilized water-in-oil microemulsions prepared in an $n$-alkane. ${ }^{52}$ At low- $Q$, the scattering data are consistent with the formation of spheres with a radius of approximately $8 \mathrm{~nm}$ and a shell thickness of $2-3 \mathrm{~nm}$. This was determined by fitting the data to a spherical form factor ${ }^{53,54}$ with a hard-sphere structure factor. ${ }^{55,56}$ The scattering is dominated by the sodium cores of the aggregates, and the thickness of the alkyl groups are simulated by varying the hard-sphere radius. The size of the polar cores is similar to that measured for AOT surfactant inverse micelles in organic solvents, ${ }^{57-59}$ although the shell thickness is larger in organic solvents compared to MMA. While unexpected, it may not be acceptable to think of a slightly polar monomer as equivalent to a highly nonpolar alkane solvent. Most importantly, these data show that AOT is self-assembled in MMA, but these aggregates do not have the same structure as in PMMA and, therefore, do not form a hexagonal template. If AOT did act as a template for hexagonal rods, a sharp peak at $Q \approx 0.3 \AA^{-1}$ would be observed.

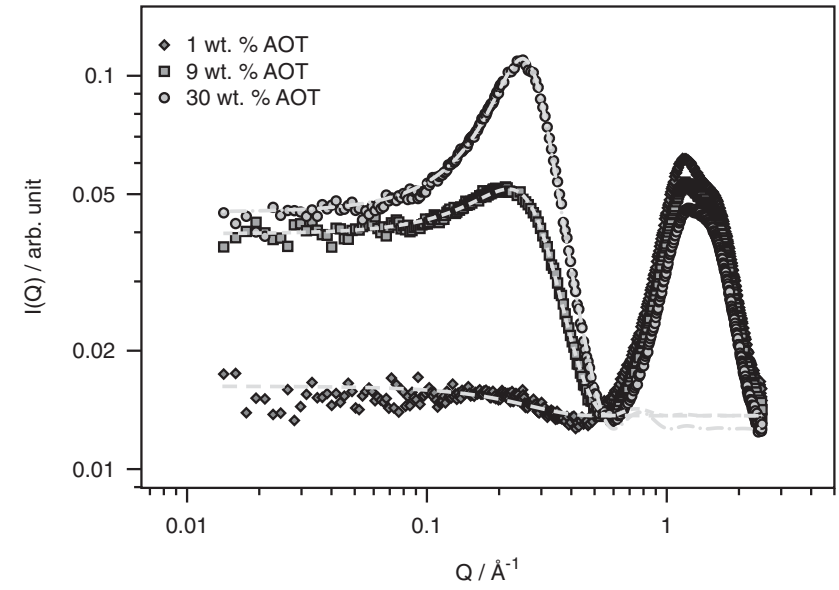

Figure 8 X-ray scattering data of AOT dispersed in MMA at several AOT concentrations. The data are well described by a spherical form factor ${ }^{53,54}$ with a hard-sphere structure factor. ${ }^{55,56}$ The radii of the spheres measured by X-ray scattering (the sodium-containing polar cores) are approximately $8 \mathrm{~nm}$ in diameter, and the thicknesses of the shells are 2-3 nm. A full colour version of this figure is available at the Polymer Journal journal online.

\section{Observations}

Considering these results as a whole shows a connection between the bulk thermal properties and the nanostructure and microstructure. The inclusion of AOT surfactant into PMMA materials has two primary bulk effects: the samples become turbid and thermally stable. The visual differences vary as a function of concentration (Figure 1); the turbidity increases proportionally to the amount of surfactant. The thermal stability appears to be independent of the amount of surfactant, over the concentration range studied here, as soon as any surfactant is incorporated the maximum degradation temperature increases by $\sim 35^{\circ} \mathrm{C}$. It is possible that there is a critical amount of AOT that is required to induce this thermal stability, but this concentration must be less than $1 \mathrm{wt}$. \%.

How does the internal structure of the PMMA-AOT composites vary as a function of concentration? Is it like the turbidity, monotonically varying with concentration, or like the thermal stability, constant once any surfactant is added? The answer depends on the lengthscale being studied. There appear to be two 'critical' concentrations. At some point below $1 \mathrm{wt}$. \% AOT, the surfactant phase seperates into species containing hexagonal rods, as determined from SAXS/MAXS/WAXS measurements (Figure 7). However, their concentration is low, corresponding to low X-ray scattering intensity (Figure 7). Although larger species must be present $\left(I(Q) \propto Q^{-4}\right.$ at low-Q), no large species containing sodium or sulfur could be observed by SEM (Figure 6). The low concentration of these species, however, likely gives rise to the turbidity seen in Figure 1 . Between 1 and 9 wt. \% AOT, the concentration of the hexagonal species increases, resulting in a more intense peak in the X-ray scattering measurements of the 9 wt. \% sample (Figure 7). This is not the only structure present in the $9 \mathrm{wt}$ \% AOT sample; $\mu \mathrm{m}$-sized aggregates, nominally spheres, as determined from SEM images and EDX measurements (Figure 6) are present. Between 9 and 30 wt. \%, the concentration of hexagonal species remains constant, but the prevalence of $\mu \mathrm{m}$-sized spheres and regions containing sodium and sulfur increases. Over this concentration range, there appear to be two types of self-assembled structure of very different sizes, with two critical concentrations for their formation. All phase separation occurs 
during the polymerization reaction, as the only self-assembled AOT species present in the MMA monomer are spheres (Figure 8).

Applying these observations, it is possible to correlate the ionic self-assembled structures with their functions. The hexagonal species are present at the lowest concentration, and the thermal stability concurrently appears along with this self-assembled AOT. This introduces a self-assembled nanostructure into the system, a type of species known to increase thermal stability, ${ }^{4-11}$ as well as structured domains of a noncombustible material. ${ }^{17}$ These properties are believed to result in the observed desirable degradation and combustion properties. The only requirement to achieve thermal stability appears to be the presence of phase separated AOT; its efficacy is not improved by increasing concentration. There are reports in the literature of sulfonates being added to polycarbonates as flame retarders, ${ }^{60-62}$ but the mechanism of activity is not clear, perhaps acting by increasing the degradation rate and forming a surface layer. ${ }^{60,61}$ In contrast, the addition of AOT increases the degradation temperature of PMMA, so the way that these two systems achieve fire retardancy does not appear to be the same. At a low concentration, AOT impacts the thermal degradation, increasing the degradation temperature, but it does not disrupt the polymerization reaction. Otherwise, the molar mass distribution would change (Figure 2), as it does for higher concentrations of AOT. At these higher concentrations, $\mu \mathrm{m}$-sized aggregates appear at the sufficiently high concentrations to cause significant turbidity. This suggests that they are responsible for the visual properties. Therefore, the lowest possible concentration of AOT would be desirable to improve the thermal properties without diminishing the optical properties.

\section{CONCLUSIONS}

The combustion resistance of PMMA-AOT composites has previously been reported ${ }^{17}$ and has been confirmed by the data presented here. In this study, the materials have been comprehensively characterized using a suite of techniques to determine the relationship between structures (from electron microscopy and X-ray scattering) and properties (from TGA and micro-cone combustion calorimetry). The temperature of maximum degradation of the composites (studied using TGA) is found to increase when AOT is incorporated; the degradation of the composites is shifted by $\sim 35^{\circ} \mathrm{C}$ compared to the homopolymer. Two parameters, the peak heat release rate and heat release capacity, are found to decrease as the amount of surfactant incorporated increases, thus indicating that the fire hazards are reduced. ${ }^{63}$ These occur without significant changes to the polymer itself. It is interesting, and potentially very useful that such improved properties can be achieved from the addition of a small concentration of a simple molecule.

By considering the structures formed by the amphiphile, AOT, the thermal stability and combustion resistance appears to be caused by the appearance of self-assembled, phase separated hexaganol AOT rods. Sodium cations and the sulfonate groups are likely to be all that remains of the self-assembled AOT when the polymer begins to degrade, as the organic groups of AOT degrade at lower temperatures $\left(\sim 300^{\circ} \mathrm{C}\right)$ than the random scission of PMMA $\left(\sim 400^{\circ} \mathrm{C}\right) .^{30,32}$ This method provides a very simple way of introducing this functional species. The sulfonates can be introduced without requiring any covalent bonds and the concentration can be varied during the sample preparation. This ease in varying the amount of surfactant means that it has been shown that only 1 wt. $\%$ of AOT needs to be introduced. From the results in this study, this seems the optimum concentration of surfactant to use. Future studies will enable further optimization of the systems; it may be possible that even lower concentrations of AOT could be used. These modifications to the formulation, however, should be straightforward due to the simplicity of the system. Given the commercial availability of the precursors and the ease in which the synthesis can be performed, these PMMA-AOT composites are interesting functional materials that can guide the preparation of more advanced thermally stable and combustion-resistant materials. Chemical links to ionic groups are not a prerequisite to thermal stability or combustion resistance, and small ionic species are a promising way to achieve this desirable property.

\section{CONFLICT OF INTEREST}

The authors declare no conflict of interest.

\section{ACKNOWLEDGEMENTS}

GNS was funded by a CASE PhD studentship (Merck Chemicals Ltd. UK, an affiliate of Merck KGaA, Darmstadt, Germany and the UK Engineering and Physical Sciences Research Council EPSRC). The Ganesha X-ray scattering apparatus used for this research was purchased under EPSRC Grant 'Atoms to Applications' Grant ref. EP/K035746/1. This work benefited from the use of the SasView application, originally developed under NSF Award DMR-0520547. SasView also contains code developed with funding from the EU Horizon 2020 programme under the SINE2020 project Grant No 654000. Mr Jonathan Jones (Electron and Scanning Probe Microscopy Facility, University of Bristol) is acknowledged for SEM images and EDX analysis.

1 Deanin, R. D. Polymer Structure, Properties and Applications (Cahners, Boston, 1972).

2 Birley, A. W. \& Scott, M. J. Plastic Materials: Properties and Applications (Leonard Hill, New York, 1982).

3 Grassie, N. \& Melville, H. W. The thermal degradation of polyvinyl compounds. II. The degradation of benzoyl peroxide catalyzed polymethyl methacrylates. Proc. $R$. Soc. Lond. A 199, 14-23 (1949).

4 Yeh, J.-M., Liou, S.-J., Lin, C.-Y., Cheng, C.-Y., Chang, Y.-W. \& Lee, K.-R. Anticorrosively enhanced PMMA-clay nanocomposite materials with quaternary alkylphosphonium salt as an intercalating agent. Chem. Mater. 14, 154-161 (2002)

5 Meneghetti, P. \& Qutubuddin, S. Synthesis, thermal properties and applications of polymer-clay nanocomposites. Thermochim. Acta 442, 74-77 (2006).

6 Konyushenko, E. N., Stejskal, J., Trchová, M., Hradil, J., Kovářová, J., Prokeš, J., Cieslar, M., Hwang, J.-Y., Chen, K.-H. \& Sapurina, I. Multi-wall carbon nanotubes coated with polyaniline. Polymer 47, 5715-5723 (2006).

7 Fukushima, K., Murariu, M., Camino, G. \& Dubois, P. Effect of expanded graphite/ layered-silicate clay on thermal, mechanical and fire retardant properties of poly (lactic acid). Polym. Degrad. Stab. 95, 1063-1076 (2010).

8 Yang, M., Zhang, S. \& Qin, H. in Physical Properties and Applications of Polymer Nanocomposites, 454-491 (Woodhead, Cambridge, 2010).

9 Kuila, T., Khanra, P., Mishra, A. K., Kim, N. H. \& Lee, J. H. Functionalized-graphene/ ethylene vinyl acetate co-polymer composites for improved mechanical and thermal properties. Polym. Test. 31, 282-289 (2012).

10 Cai, D., Jin, J., Yusoh, K., Rafiq, R. \& Song, M. High performance polyurethane/ functionalized graphene nanocomposites with improved mechanical and thermal properties. Compos. Sci. Technol. 72, 702-707 (2012).

11 Zhang, T., Xu, G., Regev, O. \& Blum, F. D. Low-temperature polymerization of methyl methacrylate emulsion gels through surfactant catalysis. J. Colloid Interface Sci. 461, 128-135 (2016)

12 Nel, A., Xia, T., Mädler, L. \& Li, N. Toxic potential of materials at the nanolevel. Science 311, 622-627 (2006).

13 Beddoes, C. M., Case, C. P. \& Briscoe, W. H. Understanding nanoparticle cellular entry: a physicochemical perspective. Adv. Colloid Interface Sci. 218, 48-68 (2015).

14 Eastoe., J. Surfactant Chemistry (Wuhan University Press, Wuhan, China, 2005).

15 Yan, F. \& Texter, J. Capturing nanoscopic length scales and structures by polymerizatioin in microemulsions. Soft Matter 2, 109-118 (2006).

16 Texter, J., Ge, L., Mourey, T. H. \& Bryan, T. G. Polymerizable bis(2-ethylhexyl) sulfosuccinate: application in microemulsion polymerization. Langmuir 20, 11288-11292 (2004).

$17 \mathrm{Ge}, \mathrm{L}$. \& Texter, J. Combustion resistant nanocomposites from water/AOT/MMA reverse microemulsions. Polym. Bull. 52, 297-305 (2004).

18 Kohlbrecher, J. \& Bressler, I. Software package SASfit for fitting small-angle scattering curves https://kur.web.psi.ch/sans1/SANSSoft/sasfit.html.

19 Breßler, I., Kohlbrecher, J. \& Thünemann, A. F. SASfit: a tool for small-angle scattering data analysis using a library of analytical expressions. J. Appl. Cryst. 48, 1587-1598 (2015)

20 Doucet, M. et al. SasView Version 4.1. Zenodo http://dx.doi.org/10.5281/ zenodo.438138. 
21 Lyon, R. E. \& Walters, R. N. Pyrolysis combustion flow calorimetry. J. Anal. Appl. Pyrolysis 71, 27-46 (2004)

22 Cogen, J. M., Lin, T. S. \& Lyon, R. E. Correlations between pyrolysis combustion flow calorimetry and conventional flammability tests with halogen-free flame retardant polyolefin compounds. Fire Mater. 33, 33-50 (2009).

23 Tyndall, J. On the blue colour of the sky, the polarization of skylight, and on the polarization of light by cloudy matter generally. Proc. R. Soc. Lond. 17, 223-233 (1868).

24 Tyndall, J. On the action of rays of high refrangibility upon gaseous matter. Phil. Trans. R. Soc. Lond. 160, 333-365 (1870).

25 Heller, W. \& Vassy, E. Tyndall spectra, their significance and application. J. Chem. Phys. 14, 565-566 (1946).

26 Heller, W., Klevens, H. B. \& Oppenheimer, H. The determination of particle sizes from Tyndall spectra. J. Chem. Phys. 14, 566-567 (1946).

27 Hiemenz, P. C. \& Lodge, T. P. Polymer Chemistry, 2nd edn (CRC Press, Boca Raton, $\mathrm{FL}, 2007)$.

28 Blum, F. D., Young, E. N., Smith, G. \& Sitton, O. C. Thermal analysis of adsorbed poly (methyl methacrylate) on silica. Langmuir 22, 4741-4744 (2006).

29 Beevers, R. B. \& White, E. F. T. Physical properties of vinyl polymers. Part 1.Dependence of the glass-transition temperature of polymethylmethacrylate on molecular weight. Trans. Faraday Soc. 56, 744-752 (1960).

30 Omastová, M., Rychlý, J., Trchová, M. \& Kovářová, J. Properties and therma decomposition of polypyrrole prepared in the presence of sodium bis(2-ethylhexyl) sulfosuccinate. Des. Monomers Polym. 7, 633-646 (2004).

31 Kissinger, H. E. Reaction kinetics in differential thermal analysis. Anal. Chem. 29, 1702-1706 (1957).

32 Ferriol, M., Gentilhomme, A., Cochez, M., Oget, N. \& Mieloszynski, J. L. Therma degradation of poly(methyl methacrylate) (PMMA): modelling of DTG and TG curves. Polym. Degrad. Stab. 79, 271-281 (2003).

33 Manring, L. E. Thermal degradation of saturated poly(methyl methacrylate). Macro molecules 21, 528-530 (1988).

34 Manring, L. E. Thermal degradation of poly(methyl methacrylate). 2. Vinyl-terminated polymer. Macromolecules 22, 2673-2677 (1989).

35 Manring, L. E., Sogah, D. Y. \& Cohen, G. M. Thermal degradation of poly(methy methacrylate). 3. Polymer with head-to-head linkages. Macromolecules 22 4652-4654 (1989).

36 Manring, L. E. Thermal degradation of poly(methyl methacrylate). 4. Random sidegroup scission. Macromolecules 24, 3304-3309 (1991).

37 Holland, B. J. \& Hay, J. N. The kinetics and mechanisms of the thermal degradation of poly(methyl methacrylate) studied by thermal analysis-Fourier transform infrared spectroscopy. Polymer 42, 4825-4835 (2001).

38 Tretsiakova-McNally, S. \& Joseph, P. Pyrolysis combustion flow calorimetry studies on some reactively modified polymers. Polymers 7, 453-467 (2015).

39 Rogers, J. \& Winsor, P. A. Change in the optic sign of the lamellar phase (G) in the Aerosol OT/water system with composition or temperature. J. Colloid Interface Sci. 30, 247-257 (1969).

40 Eastoe, J., Zou, A., Espidel, Y., Glatter, O. \& Grillo, I. Photo-labile lamellar phases. Soft Matter 4, 1215-1218 (2008).

41 Heller, W., Bhatnagar, H. L. \& Nakagaki, M. Theoretical investigations on the light scattering of spheres. XIII. The 'wavelength exponent' of differential turbidity spectra. J. Chem. Phys. 36, 1163-1170 (1962).

42 Bartoň, J., Sarov, Y. \& Capek, I. Polymerization of vinyl monomers in separated Winsor II (w/o) and Winsor I (o/w) microemulsion phases. Part 1: preparation and characterization of polymerizable vinyl-monomer-containing microemulsions. Des. Monomers Polym. 9, 153-168 (2006).

43 García, O., Sastre, R., del Agua, D., Costela, A. \& García-Moreno, I. New fluorinated polymers doped with BODIPY chromophore as highly efficient and photostable optical materials. Chem. Mater. 18, 601-602 (2006).

44 Goldstein J. I. \& Yakowitz H. (eds) Practical Scanning Electron Microscopy: Electron and Ion Microprobe Analysis (Plenum, London, 1975).

45 Jing, B., Chen, X., Zhao, Y., Wang, X., Cai, J. \& Qiu, H. Ionic self-assembled organic nanobelts from the hexagonal phase complexes and their cyclodextrin inclusions. J. Phys. Chem. B 112, 7191-7195 (2008).

46 Lai, W.-C. A method for preparing self-assembled gels from opaque solutions with high concentrations of AOT. Colloids Surf. A: Physicochem. Eng. Aspects 433, 139-144 (2013).

47 Camerel, F., Strauch, P., Antonietti, M. \& Faul, C. F. J. Copper-metallomesogen structures obtained by ionic self-assembly (ISA): Molecular electromechanical switching driven by cooperativity. Chem. Eur. J. 9, 3764-3771 (2003).

48 Grillo, I. in Soft Matter Characterization (eds R. Borsali and R. Pecora) 723-782 (Springer, the Netherlands, 2008).

49 Lovell, R. \& Windle, A. H. Determination of the local conformation of PMMA from wideangle X-ray scattering. Polymer 22, 175-184 (1981).

50 Hammouda, B. Probing nanoscale structures-The SANS toolbox.

51 Skouri, M., Marignan, J. \& May, R. X-ray and neutron-scattering study of the lamellar and $\mathrm{L}_{3}$ phases of the system aerosol-OT-water: effect of $\mathrm{NaCl}$ and decane. Colloid Polym. Sci. 269, 929-937 https://www.ncnr.nist.gov/staff/hammouda/ (1991).

52 Calandra, P., Longo, A., Marcianò, V. \& Liveri, V. T. Physicochemical investigation of lightfast $\mathrm{AgCl}$ and $\mathrm{AgBr}$ nanoparticles synthesized by a novel solid-solid reaction. J. Phys. Chem. B 107, 6724-6729 (2003).

53 Rayleigh, $L$. The incidence of light upon a transparent sphere of dimensions comparable with the wave-length. Proc. R. Soc. Lond. A 84, 25-46 (1910).

54 Guinier, A. \& Fournet, G. Small-Angle Scattering of X-Rays (John Wiley \& Sons, New York, 1955).

55 Percus, J. K. \& Yevick, G. J. Analysis of classical statistical mechanics by means of collective coordinates. Phys. Rev. 110, 1-13 (1958).

56 Vrij, A. Mixtures of hard spheres in the Percus-Yevick approximation. Light scattering at finite angles. J. Chem. Phys. 71, 3267-3270 (1979).

57 Kotlarchyk, M., Huang, J. S. \& Chen, S.-H. Structure of AOT reversed micelles determined by small-angle neutron scattering. J. Phys. Chem. 89, 4382-4386 (1985).

58 Smith, G. N., Brown, P., Rogers, S. E. \& Eastoe, J. Evidence for a critical micelle concentration of surfactants in hydrocarbon solvents. Langmuir 29 3252-3258 (2013)

59 Smith, G. N., Brown, P., James, C., Kemp, R., Khan, A. M., Plivelic, T. S., Rogers, S. E. \& Eastoe., J. The effects of counterion exchange on charge stabilization for anionic surfactants in nonpolar solvents. J. Colloid Interface Sci. 465, 316-322 (2016).

60 Wang, Y.-Z., Yi, B., Wu, B., Yang, B. \& Liu, Y. Thermal behaviors of flame-retardant polycarbonates containing diphenyl sulfonate and poly(sulfonyl phenylene phosphonate). J. Appl. Polym. Sci. 89, 882-889 (2003).

61 Levchik, S. V. \& Weil, E. D. Overview of recent developments in the flame retardancy of polycarbonates. Polym. Int. 54, 981-998 (2005).

62 Levchik, S. V. \& Weil, E. D. Flame retardants in commercial use or in advanced development in polycarbonates and polycarbonate blends. J. Fire Sci. 24 137-151 (2006).

63 Babrauskas, V. \& Peacock, R. D. Heat release rate: the single most important variable in fire hazard. Fire Saf. J. 18, 255-272 (1992).

Supplementary Information accompanies the paper on Polymer Journal website (http://www.nature.com/pj) 